\section{Endocrinology: Times Change}

\author{
Kostas Markou* \\ Department of Internal Medicine, University of Patras Medical School,
} Greece

\section{Introduction}

The rapid developments in the field of medical sciences as well as science as a whole, have paved the way for the production of a vast load of information that can be very difficult to evaluate. The study of the human physiology as well as the "physiology" of the mechanisms observed in the society has revealed that these two share common properties.

The complexity of the human body requires means of communication between the cells, organs and systems. In the greater part, this communication is achieved through the Endocrine and Neural systems that constitute the "telecommunications" system of the human body. A pertinent parallelism with telecommunications is as follows: the neural system represents the old fashioned telephone communication and the endocrine system the mobile communication. Based on the above parallel, the neural system can convey messages to the endocrine just like the landline can call a mobile phone and vice versa. Even more for the endocrine system, this parallel can be further extended to modern technologies since the endocrine glands secretion can target either the same secretory cell (autocrine function-single user PC) or adjacent cells (paracrine function-LAN (Local Area Network)) or even tissues all over the body (endocrine function-internet). Bearing in mind that humans are inspired by nature and to a great extent try to imitate it, the significant similarities between the message transmission systems of the human body and human inventions should not come as a surprise. On the contrary, the exemplary function of the endocrine system forces us to accept the fact that nature's telecommunications messages will not be deciphered for many years to come.

Across the entire spectrum, from single cell organisms to the human, an inner clock exists which is called circadian (circa diem). This circadian rhythm is driven by endogenous stimulants and has a

*Corresponding author: Kostas Markou, Department of Internal Medicine University of Patras Medical School, Panepistimioupoli Patron 265 04, Greece, Tel: +30 2610 999732; E-mail: markoukonst@upatras.gr

Citation: Markou K (2016) Endocrinology: Times Change. J Hum Endocrino 1: 001.

Received: February 29, 2016; Accepted: March 07, 2016; Published: March 21, 2016

Copyright: (C) 2016 Markou K, This is an open-access article distributed under the terms of the Creative Commons Attribution License, which permits unrestricted use, distribution, and reproduction in any medium, provided the original author and source are credited. period of 24 hours. This daily rhythm is responsible for the stability of the metabolism and the energy production needed for the various functions of the body. Moreover, the rhythm prepares the body for the daily changes of the ambient conditions. The diurnal rhythm of the endocrine system allows also the optimal acquisition and exploitation of the available energy, specifically in plants by the introduction of the light-dark cycle and in animals and humans by implementation of the sleep-wake cycle. This relation is an indicator of the highly important connection between circadian rhythm and metabolic system. In fact, there are two clocks, a central clock in the hypothalamus and a peripheral in other organs such as fat tissue, liver and muscles. Light is the stimulus of the central clock while food intake is the stimulus of the peripheral [1].

In the endocrine system, specialized organs, the glands, secrete specific substances, the hormones, and convey messages to other organs and cells. The various cells must be equipped with appropriate receptors in order to receive the message. The endocrinology cannot be defined by strict anatomic terms. The "classic" endocrine glands (pituitary, thyroid, parathyroid, pancreatic islet cells, adrenals and gonads) communicate with other organs in a complex manner via neural system, hormones, cytokines and growth factors. Through the production of the hypothalamic stimulatory factors, the central neural system holds a significant role in the regulation of the pituitary hormone secretion. However, substances with endocrine function are not secreted only but the glands mentioned above but also by other organs. The heart is the main producer of atrial natriuretic peptide which in turn acts, in a classical endocrine manner, on the kidney and provokes natriuresis. The kidney produces erythropoietin that stimulates the red blood cell production, while the gastrointestinal system produces various peptide hormones such as ghrelin, holocystokine, gastrin, secretin, Vasoactive Intestine Peptide (VIP) [2].

The Journal of Human Endocrinology aspires to serve the ever increasing demand for reliable information on both basic and clinical research. As of today, the endocrine community has yet another ally in the effort to promote and expand science.

\section{References}

1. Dominoni DM, Borniger JC, Nelson RJ (2016) Light at night, clocks and health: from humans to wild organisms. Bio Lett 12.

2. http://www.endotext.org/ 